\section{Características maternas em gestações com risco de prematuridade tardia}

\section{Maternal characteristics in pregnancies at risk for late preterm childbirth}

\begin{abstract}
Objectives: to evaluate maternal characteristics in cases of late prematurity.

Methods: we carried out a cross-sectional study with secondary analysis of pregnant women admitted to a randomized clinical trial conducted in Northeast of Brazil from 2008 to 2010, between 34 and 36 weeks. We analyzed the maternal variables: age, parity, gestational age, history of prematurity and associated medical conditions. Statistical analysis was performed using Epi-Info 3.5.3, with distribution of frequency tables for categorical variables and central tendency and dispersion measures for numerical. Chi-square test was used to determine the association between frequency of morbidities by stratum of gestational age. A significance level of $5 \%$ was adopted.
\end{abstract}

Results: a total of 273 women in IMIP with conditions leading to imminent risk of late preterm births. The mean age was 23.1 years and $28.6 \%$ were teenagers and primipara were $53.8 \%$. The mean gestational age was 35 weeks. About $12 \%$ had a history of prematurity. The conditions associated with late prematurity were: preterm labor (67\%), ruptured membranes (39.6\%), hypertensive disorders (25.3\%). There was no difference in frequency of these conditions by subgroups of gestational age.

Conclusions: spontaneous preterm labor, ruptured membranes and hypertensive disorders were the most common clinical conditions associated with late prematurity.

Key words Pregnancy complications, Premature birth, Morbidity

\author{
Ana Maria Feitosa Porto 1 \\ Débora Ávila Acioly 2 \\ Isabela Coutinho 3 \\ Eduardo Henrique Correa Coutinho 4 \\ Paula Silva Bezerra 5 \\ Melania Maria Ramos de Amorim 6
}

\begin{abstract}
1-6 Instituto de Medicina Integral Prof. Fernando Figueira (IMIP). Rua dos Coelhos, 300. Boa Vista. Recife, PE, Brasil. CEP: 50.070-550. E-mail:melamorim@uol.com.br
\end{abstract}

\section{Resumo}

Objetivos: avaliar as características maternas na prematuridade tardia.

Métodos: foi realizado um estudo do tipo corte transversal, com análise secundária das gestantes incluidas em um ensaio clínico randomizado realizado no Nordeste do Brasil, no periodo de 2008 a 2010, entre 34 e 36 semanas. Analisadas as variáveis maternas: idade, paridade, idade gestacional, antecedentes de prematuridade e condições clínicas associadas. A análise estatística foi realizada no programa Epi-Info 3.5.3. Foram construidas tabelas de distribuição de frequência para as variáveis categóricas e calculadas medidas de tendência central e de dispersão para as numéricas. Para determinação da associação entre a frequência das morbidades por estratos de idade gestacional, utilizou-se o teste qui-quadrado de associação. Considerou-se o nível de significância de 5\%.

Resultados: foram analisadas 273 mulheres no IMIP com risco iminente de prematuridade tardia. A idade média foi 23,1 anos, 28,6\% eram adolescentes e 53,8\% primíparas. A idade gestacional média foi 35 semanas. Cerca de $12 \%$ tinham antecedentes de prematuridade. As condições associadas à prematuridade tardia foram: trabalho de parto prematuro (67\%), ruptura prematura das membranas (39,6\%), sindromes hipertensivas (25,3\%), sem haver diferença da frequência por subgrupos de idade gestacional.

Conclusões: trabalho de parto prematuro espontâneo, ruptura prematura das membranas e sindromes hipertensivas foram as condições clinicas mais frequentemente associadas com prematuridade tardia. Palavras-chave Complicações na gravidez, Nascimento prematuro, Morbidade 


\section{Introdução}

Segundo o conceito da Organização Mundial da Saúde (OMS 1972), acatado pela American Academy of Pediatrics e pelo American College of Obstetricans and Gynecologists, o parto pré-termo é definido por nascimento que ocorre antes de 37 semanas completas baseadas no início do último período menstrual. ${ }^{1}$

Em julho de 2005, o National Institute of Child Health and Human Development (NICHD) promoveu um workshop recomendando o uso do termo "pré-termo tardio" para aqueles recémnascidos entre a $34^{\mathrm{a}}$ semana $(340 / 7)$ e a $36^{\mathrm{a}}$ semana completa de gestação $\left(36^{6 / 7}\right) .^{2}$

Durante muito tempo acreditou-se que lactentes pré-termo tardios seriam fisiologicamente e metabolicamente tão maduros quanto um lactente a termo. No entanto, estudos recentes mostram que, quando comparados à recém-nascidos a termo, os prematuros tardios têm risco elevado para desenvolver complicações, incluindo distúrbios respiratórios, hiperbilirrubinemia, instabilidade na temperatura, problemas com a alimentação, hipoglicemia, resultando em internamento prolongado e maior frequência de readmissão hospitalar no primeiro mês de vida.3,4

As condições obstétricas mais frequentemente associadas ao parto pré-termo tardio são: ruptura prematura das membranas, gestações múltiplas, trabalho de parto prematuro espontâneo 5 e indicações médicas para interrupção da gestação (representadas principalmente por pré-eclâmpsia e restrição do crescimento fetal). 6

É importante identificar e avaliar gestantes com essas condições, visando delinear estratégias para prevenir a prematuridade tardia e suas consequências. Pela morbidade neonatal a curto e a longo prazo e os custos associados, os cuidados obstétricos deveriam ter como foco a real necessidade do parto neste período da gestação, que deveria ficar restrito aos casos de indiscutível indicação.7

Portanto, diante das evidências de que nascimentos entre a $34^{\mathrm{a}}$ e a $36^{\mathrm{a}}$ semana de gestação determinam maior morbidade e mortalidade para o recém-nascido, bem como elevados custos financeiros ao sistema de saúde quando comparados aos de termo, realizou-se o presente estudo com o objetivo de avaliar as características maternas nos casos de prematuridade tardia atendidos em um hospitalescola do Nordeste do Brasil.

\section{Métodos}

Foi realizado um estudo transversal sendo incluídas gestantes com risco iminente de prematuridade tardia que participaram de um ensaio clínico conduzido no Instituto de Medicina Integral Prof. Fernando Figueira (IMIP) em Recife, Pernambuco, com o objetivo de avaliar a efetividade da corticoterapia antenatal entre a $34^{\mathrm{a}}$ e a $36^{\mathrm{a}}$ semana para redução dos distúrbios respiratórios nos recémnascidos. O estudo original incluiu 320 gestantes com idade gestacional entre a $34^{\mathrm{a}}$ e a $36^{\mathrm{a}}$ semana. Foram excluídos desta análise os casos que chegaram a termo e as mulheres que tiveram alta ainda gestantes, desconhecendo-se os resultados do parto por ter sido assistido em outros serviços. Desta forma, foram analisadas as características de 273 gestantes.

O estudo foi realizado no período entre maio de 2008 e julho de 2010, tendo sido previamente aprovado pelo Comitê de Ética em Pesquisa da Instituição (Parecer $n^{\circ}$ 1029) e registrado na plataforma ClinicalTrials.gov sob o número NCT00675246.8 Todas as gestantes incluídas concordaram em participar e assinaram o Termo de Consentimento Livre e Esclarecido.

Foram incluídas gestantes com idade gestacional entre 34 e 36 semanas, com diagnóstico de iminência de parto prematuro espontâneo ou com indicação médica (materna e/ou fetal) de antecipação do parto. Foram excluídos os casos de gestação múltipla, malformações congênitas, previsão de interrupção imediata da gestação por indicação materna ou fetal, síndromes hemorrágicas, evidência clínica de corioamnionite e uso prévio de corticoterapia.

As variáveis analisadas foram idade da gestante (frequência de adolescentes e mulheres com idade maior ou igual a 35 anos), paridade (percentual de primíparas), idade gestacional na admissão no estudo (da $34^{\mathrm{a}}$ a $36^{\mathrm{a}}$ semana), antecedentes de prematuridade (história de pelo menos um parto prematuro anterior) e morbidades maternas associadas.

A idade gestacional foi avaliada pela data do último período menstrual, desde que conhecida e confiável, ou por ultrassonografia realizada antes da $20^{\text {a }}$ semana. Definiu-se como prematuridade tardia a ocorrência do parto prematuro entre a $34^{\mathrm{a}}$ semana de gestação (340/7 ou 238 dias desde o primeiro dia do último período menstrual) e a $36^{\mathrm{a}}$ semana completa (366/7 ou 258 dias desde o primeiro dia da última menstruação). 2

As morbidades maternas consideradas foram aquelas diagnosticadas na admissão, antes da 
inclusão no estudo: síndromes hipertensivas, ruptura prematura das membranas, trabalho de parto prematuro, oligo-hidrâmnio, restrição do crescimento fetal e diabetes. Síndromes hipertensivas foram definidas pelos critérios propostos pelo National High Blood Pressure Working Education Program (2000). ${ }^{9}$ Ruptura prematura das membranas foi definida pela ruptura da bolsa das águas antes de iniciado o trabalho de parto e trabalho de parto prematuro foi caracterizado pela presença de contrações dolorosas e palpáveis com frequência de pelo menos quatro em cada 30 minutos com duração de 30 segundos em gestação abaixo de 37 semanas, associado a modificações na posição, consistência, comprimento e/ou dilatação do colo uterino. ${ }^{10}$

Considerou-se como diabetes tanto o diabetes clínico quanto o gestacional, definido por qualquer grau de intolerância à glicose com início ou reconhecimento durante a gravidez.11,12 Oligo-hidrâmio foi definido por ultrassonografia como um índice de líquido amniótico (ILA) abaixo de 5,013 e restrição do crescimento fetal como a presença de fetos abaixo do percentil 10 da curva de crescimento ultrassonográfica. 14

A análise estatística foi realizada utilizando-se o programa Epi-Info versão 3.5.1. Foram construídas tabelas de distribuição de frequência para as variáveis categóricas e calculadas medidas de tendência central e de dispersão para as variáveis numéricas. Para determinação da associação entre a frequência das diversas morbidades por estratos de idade gestacional, utilizou-se o teste qui-quadrado de associação, considerando-se o nível de significância de 5\%.

\section{Resultados}

Neste estudo a média de idade das gestantes foi de 23,1 anos com desvio padrão de $\pm 5,8$, sendo $28,6 \%$ delas com menos de 20 anos e 4,8\% com 35 anos ou mais. A paridade variou entre zero e nove, com mediana de zero. Houve 147 primíparas, representando um percentual de $53,8 \%$ das gestantes admitidas. A idade gestacional média foi de 35,0 semanas com um desvio padrão de $\pm 0,7$. Cerca de metade das pacientes estava na $35^{\mathrm{a}}$ semana de gestação, aproximadamente $25 \%$ na $34^{\mathrm{a}}$ e $25 \%$ na $36^{\mathrm{a}}$ semana. Quase $12 \%$ tinham antecedente de prematuridade anterior (Tabela 1).

\begin{tabular}{|c|c|c|}
\hline Características & $\mathbf{N}$ & $\%$ \\
\hline Idade em anos ( $\bar{X} \pm D P),($ min-máx $)$ & & $23,1(5,83),(12-41)$ \\
\hline Idade menor que 20 anos $(n, \%)$ & 78 & 28,6 \\
\hline Idade maior ou igual a 35 anos (n, \%) & 13 & 4,8 \\
\hline Paridade (mediana) (min-máx) & & $0(0-9)$ \\
\hline Primíparas (n, \%) & 147 & 53,8 \\
\hline Idade gestacional ( $\bar{X} \pm D P),($ min-máx $)$ & & $35+0,7,(34-36)$ \\
\hline 34 semanas & 69 & 25,3 \\
\hline 35 semanas & 137 & 50,2 \\
\hline 36 semanas & 67 & 24,5 \\
\hline Antecedentes de prematuridade (n, \%) & 32 & 11,7 \\
\hline
\end{tabular}

Em relação às condições clínicas associadas, o trabalho de parto prematuro foi a mais prevalente, representando um percentual de $67 \% \quad(183$ pacientes). A segunda condição clínica mais frequente foi a ruptura prematura das membranas, com um percentual de 39,6\% (108 pacientes). As síndromes hipertensivas tiveram uma frequência de $25,3 \%$ (69 casos) entre as gestantes analisadas. As pacientes com diagnóstico de oligo-hidrâmnio tiveram uma frequência de 7,3\% (20 pacientes) e apenas três pacientes $(1,1 \%)$ apresentavam associação com restrição do crescimento fetal. Diabetes foi uma condição clínica pouco frequente, com um percentual de apenas $1,8 \%$ (cinco casos) (Tabela 2).

Analisando as condições clínicas por subgrupo de idade gestacional, o trabalho de parto prematuro teve uma frequência variando de $65,2 \%$ a $68,6 \%$ nas três semanas avaliadas. Ruptura prematura das 
membranas foi encontrada em 33,3\%, 41,6\%, 41,8\% das gestantes na $34^{\mathrm{a}}, 35^{\mathrm{a}}$ e $36^{\mathrm{a}}$ semanas, respectivamente. Síndromes hipertensivas foram encontradas em $24,6 \%, 23,4 \%$ e $29,9 \%$ na $34^{a}, 35^{a}$ e $36^{a}$ semana, respectivamente. Não houve diferença significativa, exceto para oligo-hidrâmnio $(p<0,02)$ na frequência das condições clínicas analisadas por idade gestacional entre a $34^{\mathrm{a}}$ e a $36^{\mathrm{a}}$ semana, com taxas semelhantes de gestantes com síndromes hipertensivas, ruptura prematura das membranas, trabalho de parto prematuro, restrição do crescimento fetal e diabetes (Tabela 3).

Tabela 2

Principais condições clínicas presentes em gestantes com risco de prematuridade tardia.

\begin{tabular}{lcc}
\hline Condições clínicas associadas & N & $\%$ \\
\hline Trabalho de parto prematuro & 183 & 67,0 \\
Ruptura prematura das membranas & 108 & 39,6 \\
Síndromes hipertensivas & 69 & 25,3 \\
Oligodrâmnio & 20 & 7,3 \\
Diabetes & 5 & 1,8 \\
Restrição do crescimento fetal & 3 & 1,1 \\
\hline
\end{tabular}

Tabela 3

Condições clínicas maternas associadas por subgrupos de idade gestacional entre a $34^{\mathrm{a}}$ e a $36^{\mathrm{a}}$ semana.

\begin{tabular}{|c|c|c|c|c|c|c|c|}
\hline \multirow{3}{*}{ Condições clínicas } & \multicolumn{6}{|c|}{ Idade gestacional } & \multirow{3}{*}{$p$} \\
\hline & \multicolumn{2}{|c|}{34 semanas } & \multicolumn{2}{|c|}{35 semanas } & \multicolumn{2}{|c|}{36 semanas } & \\
\hline & $\mathrm{n}$ & $\%$ & $\mathrm{n}$ & $\%$ & $\mathrm{n}$ & $\%$ & \\
\hline Trabalho de parto prematuro & 17 & 24,6 & 32 & 23,4 & 20 & 29,9 & 0,60 \\
\hline Ruptura prematura das membranas & 23 & 33,3 & 57 & 41,6 & 28 & 41,8 & 0,50 \\
\hline Síndromes hipertensivas & 10 & 14,5 & 5 & 3,6 & 5 & 7,5 & 0,02 \\
\hline Oligodrâmnio & 45 & 65,2 & 94 & 68,6 & 44 & 65,7 & 0,80 \\
\hline Diabetes & 2 & 2,9 & 1 & 0,7 & 0 & 0,0 & 0,20 \\
\hline Restrição do crescimento fetal & 2 & 2,9 & 2 & 1,5 & 2 & 1,5 & 0,70 \\
\hline
\end{tabular}

\section{Discussão}

O presente estudo permite avaliar as principais características encontradas em gestações únicas com risco iminente de prematuridade tardia, excetuandose as emergências que representaram critério de exclusão para o estudo original.

Diversos estudos têm sido publicados sobre os recém-nascidos pré-termo tardios e está bem demonstrado o aumento da morbidade e mortalidade dessa população. 15,16

A maior parte das gestantes com risco de prematuridade tardia concentrava-se na faixa de idade gestacional da $35^{\mathrm{a}}$ semana, correspondendo à metade dos casos, distribuindo-se simetricamente os extremos (25\%) com 34 e 36 semanas, o que resultou em uma média de idade gestacional em torno de 35 semanas na admissão. Os estudos demonstram que recémnascidos com essa idade gestacional têm morbidade relevante e, portanto, a conduta obstétrica nessas situações deve levar em consideração os riscos neonatais que podem advir do nascimento. 17

Observamos que a média de idade foi em torno de 23 anos, com uma frequência de adolescentes em torno de $29 \%$ e quase $5 \%$ de mulheres com 35 anos ou mais. Essas características, porém, são semelhantes às encontradas na população obstétrica geral de nosso serviço e não servem como discriminante para identificar gestantes de risco para prematuri- 
dade tardia.

Em relação à paridade, foi elevada uma frequência de primíparas em torno de $54 \%$ e antecedentes de prematuridade em apenas $12 \%$ das mulheres. Desta forma, tanto primíparas como multíparas podem se encontrar em situação de risco para prematuridade tardia, não discriminada pela paridade ou pelo antecedente de parto prematuro anterior. Deve-se, contudo, ressaltar que história de parto prematuro anterior representa o melhor preditor clínico isolado do parto prematuro entre multíparas, com recorrência em torno de $16 \%{ }^{18}$

$\mathrm{O}$ achado mais relevante deste estudo refere-se à importante presença de diversas condições clínicas na gravidez, as quais sobrelevam o risco de prematuridade tardia. Encontramos $67 \%$ de casos de trabalho de parto prematuro, $39,6 \%$ de ruptura prematura das membranas e $25,3 \%$ de síndromes hipertensivas, o que é semelhante ao que já foi descrito por outros autores analisando as causas maternas de prematuridade tardia.5,17

Diversos outros estudos têm demonstrado, como o nosso, que o trabalho de parto prematuro espontâneo representa a principal causa de prematuridade tardia, tendo sido observado que se encontra em ascensão nessa população específica. ${ }^{6}$ Possivelmente este fato tem relação com a prática habitual de se realizar tocólise e corticoterapia apenas em gestantes com trabalho de parto com menos de 34 semanas, que representa o grupo onde se observa maior benefício com essas estratégias para prevenção de distúrbios respiratórios e outras morbidades. 19 Como apenas recentemente os recém-nascidos pré-termo tardios têm sido reconhecidos como uma população de risco para morbidade e mortalidade neonatal,16 diversos profissionais e centros não têm adotado a prática de inibir o trabalho de parto prematuro depois de 34 semanas, o que contribui para um aumento na frequência de prematuros tardios. ${ }^{17}$

Da mesma forma, a ruptura prematura das membranas, segunda causa mais frequente de prematuridade em nosso estudo, também tem apresentado frequência em ascensão nas últimas décadas, na idade gestacional entre a $34^{\mathrm{a}}$ e a $37^{\mathrm{a}}$ semana. ${ }^{6}$ Existem controvérsias em relação à conduta quando ocorre ruptura da bolsa das águas nesse período e a recomendação de experts é de conduta conservadora apenas em gestações com menos de 34 semanas, ${ }^{20} \mathrm{o}$ que também contribui para elevar o número de nascimentos pré-termo tardios. O conhecimento do impacto desses nascimentos na saúde pública é importante para uma melhor discussão sobre a adoção de uma conduta ativa versus uma conduta expectante nesta situação. 21
Em relação às síndromes hipertensivas, que representaram a terceira causa de prematuridade tardia no nosso estudo, os achados da literatura são discrepantes e não está ainda elucidado o seu real papel no contexto da prematuridade tardia.22,23 Mesmo assim, é relevante que tanto hipertensão arterial crônica como pré-eclâmpsia respondam por $25 \%$ das causas maternas de prematuridade tardia, o que é compatível com a elevada taxa de hipertensão na gravidez em nosso meio e também com a expressiva contribuição das síndromes hipertensivas na gênese da prematuridade global. Efetivamente, o parto prematuro terapêutico é amiúde indicado para a resolução dos quadros de pré-eclâmpsia, uma vez que a única cura possível para esse transtorno é o parto. Na maioria dos protocolos de manejo dos casos graves de pré-eclâmpsia, prevê-se a interrupção da gravidez depois da $34^{\mathrm{a}}$ semana21-23 e portanto, é possível que esta condição também seja uma causa relevante de prematuridade tardia em todo o mundo.

Destacamos que a não existência de diferença significativa na distribuição desses principais distúrbios (trabalho de parto prematuro, ruptura prematura das membranas e síndromes hipertensivas) por subgrupos de idade gestacional, indica que sua importância como causas de prematuridade tardia independe da fase da gravidez, exceto a oligohidramnia onde houve significância estatística, com $p<0,02$, sendo encontrados $14,5 \%$ na $34^{\mathrm{a}}$ semana, $3,6 \%$ na $35^{\mathrm{a}}$ semana e $7,5 \%$ na $36^{\mathrm{a}}$ semana de gestação. Este achado com frequência maior na $34^{\mathrm{a}}$ semana, não encontra concordância com a literatura, uma vez que o líquido amniótico diminui com a idade gestacional e seu achado isolado, não deveria ser indicação da antecipação do parto antes de 37 semanas. ${ }^{24}$ No nosso estudo é possível ser um achado associado a outras entidades clínicas, como as síndromes hipertensivas.

O presente estudo tem limitações por se tratar de uma análise secundária de um ensaio clínico realizado com o objetivo de avaliar a efetividade da corticoterapia antenatal em gestantes com risco iminente de prematuridade tardia. Foram, portanto, excluídos os casos de gestação gemelar, que representa outra causa importante de prematuridade tardia, além das situações de emergência acarretando necessidade de imediata interrupção da gravidez. No entanto, as causas de prematuridade tardia aqui encontradas equivalem à maior parte das causas descritas na literatura.5,17 A exclusão das emergências possivelmente não tem grande impacto, uma vez que em uma situação de emergência, como eclâmpsia ou placenta prévia com hemorragia rele- 
vante, é impossível a adoção de medidas preventivas como a postergação do parto, uso de tocolíticos ou administração de corticóide, estratégias que devem ser discutidas em todas as outras situações aqui encontradas.

Postergar o parto pode ser a principal medida para prolongar a gravidez, aumentar peso e idade gestacional e assim, evitar uma frequência elevada de morbidade neonatal, sobretudo de distúrbios respiratórios. 21,23 Configura-se um delicado balanço em que os riscos de um parto prematuro devem ser pesados contra os riscos de um ambiente intrauterino

\section{Referências}

1. Engle WA. A recommendation for the definition of "Late Preterm" (Near-Term) and the birth weight-gestational age classification system. Semin Perinatol. 2006; 30: 2-7.

2. Raju TNK. Epidemiology of late preterm (near-term) births. Clin Perinatol. 2006; 33: 751-63.

3. Yoder BA, Gordon MC, Barth Jr WH. Late-preterm birth Obstet Gynecol. 2008; 111: 814-22.

4. Ramachandrappa A, Jain L. Health issues of the late preterm infant. Pediatr Clin North Am 2009; 56: 565-77.

5. Dobak WJ, Gardner MO. Late preterm gestation: physiology of labor and implications for delivery. Clin Perinatol. 2006; 33: 765-76.

6. Davidoff MJ, Dias T, Damus K, Russell R, Bettegowda VR, Dolan S, Schwarz RH, Green NS, Petrini J. Changes in the gestational age distribution among U. S. singleton birth: impact on rates of late preterm birth, 1992 to 2002. Semin Perinatol. 2006; 30: 8-15.

7. Loftin RW, Habli M, Snyder CC, Cormier CM, Lewis DF, DeFranco EA. Late preterm birth. Obstet Gynecol. 2010; 3: 10-9.

8. Porto AM, Coutinho IC, Correia JB, Amorim MM Effectiveness of antenatal corticosteroids in reducing respiratory disorders in late preterm infants: randomised clinical trial. BMJ 2011; 342: d1696.

9. National High Blood Pressure Education Program Working Group. Report on high blood pressure in pregnancy. Am J Obstet Gynecol. 2000; 183: S1-S22.

10. Lamont RF and the International Preterm Labour Council. Evidence-based labour ward guidelines for the diagnosis, management and treatment of spontaneous preterm labour. J Obstet Gynaecol. 2003; 23: 469-78.

11. Carpenter MW, Coustan DR. Criteria for screening tests for gestational diabetes. Am J Obstet Gynecol. 1982; 144: 76873.

12. American Diabetes Association. Standards of medical care in diabetes - 2009. Diabetes Care. 2009; 32 (Suppl. 1): S13-61. adverso, o que aumentaria as chances de hipóxia perinatal e baixos escores de Apgar ao nascimento. No entanto, na presença de vitalidade fetal satisfatória, acreditamos que se deva considerar a possibilidade de estender os limites de idade gestacional para antecipação do parto em gestações de alto-risco, sobretudo em casos de ruptura prematura das membranas e nas síndromes hipertensivas. O papel dos tocolíticos para postergar o parto na vigência de trabalho de parto depois de 34 semanas também precisa ser estabelecido.
13. Phelan JP, Smith CV, Broussard P, Small M. Amnitic fluid volume assessment with the four-quadrant thecnique at 3642 week's gestation. J Reprod Med. 1987; 32: 540-2.

14. Cecatti JG, Machado MRM, Santos FFA, Marussi EF. Curva dos valores normais de peso fetal estimado por ultrassonografia segundo a idade gestacional. Cad Saúde Pública. 2000; 16: 1083-90.

15. Raju TNK, Higgins RD, Stark AR, Leveno KJ. Optimizing care and outcome for late-preterm (near-term) infants: a summary of the workshop sponsored by the National Institute of Child Health and Human Development. Pediatrics. 2006; 118: 1207-13.

16. Wang ML, Dorer DJ, Fleming MP, Catlin EA. Clinical outcomes of near-term infants. Pediatrics. 2004; 114: 3726.

17. Fuchs K, Wapner R. Eletive cesarean section and induction and their impact on late preterm births. Clin Perinatol. 2006; 33: 793-801.

18. Cunningham FG, Leveno KJ, Bloom SL, Hauth JC, Gilstrap III LC, Wenstrom KD. Williams Obstetrics. 22 ed. New York: McGraw-Hill; 2005

19. Roberts D, Dalziel S. Antenatal corticosteroids for accelerating fetal lung maturation for women at risk of preterm birth. Cochrane Database Syst Rev. 2006; 19: CD004454.

20. Mercer BM. Preterm premature rupture of the membranes. Obstet Gynecol. 2003; 101: 178-93.

21. Fuchs K, Gyamfi C. The influence of obstetric practices on late prematurity. Clin Perinatol. 2008; 35: 343-60

22. Sibai BM. Preeclampsia as a cause of preterm and late preterm (near-term) births. Semin Perinatol. 2006; 30: 169.

23. Kominiarek MA. Infants born late preterm: indications and recommendations for obstetric care. NeoReviews. 2009; 10 : e295-302.

24. Spong CY, Mercer BM, D'Alton M, Kilpatrick S, Blackwell S, Saade G. Timing of indicated late-preterm and early-term birth. Obstet Gynecol. 2011; 118: 323-333.

Recebido em 13 de abril de 2011

Versão final apresentada em 4 de março de 2013

Aprovado em 3 de abril de 2013 